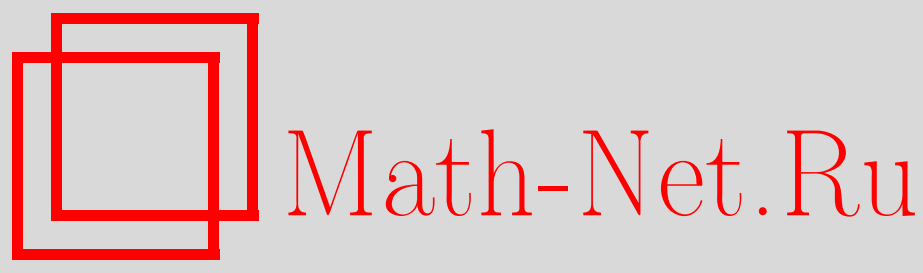

В. В. Жаринов, Эволюционные системы на решетке, ТМФ, 2008, том 157, номер 3, 391-405

DOI: https://doi.org/10.4213/tmf6287

Использование Общероссийского математического портала Math-Net.Ru подразумевает, что вы прочитали и согласны с пользовательским соглашением http://www . mathnet.ru/rus/agreement

Параметры загрузки:

IP: 54.198 .187 .58

26 апреля 2023 г., 06:21:57

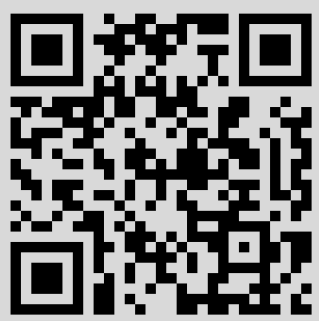




\section{ЭВОЛЮЦИОННЫЕ СИСТЕМЫ НА РЕШЕТКЕ}

В рамках алгебро-геометрического подхода к дифференциально-разностным уравнениям изучаются симметрии и законы сохранения эволюционных систем на многомерных решетках. Дано описание законов сохранения в терминах их характеристик.

Ключевые слова: многомерная целочисленная решетка, разностные джеты, симметрии, законы сохранения, характеристики законов сохранения.

В настоящей работе результаты, полученные ранее [1] для эволюционных систем на евклидовом пространстве, переносятся на эволюционные системы на многомерной целочисленной решетке. С этой целью основные принципы алгебро-геометрического (иначе, группового) анализа уравнений в частных производных (см., например, [2]-[6]) адаптируются к дискретной ситуации. Вводится понятие разностного джета, изучаются функции на пространстве разностных джетов и операции над ними, приводятся элементы вариационного исчисления на решетке. Развитый таким образом формализм применяется для описания законов сохранения эволюционных систем на многомерной решетке в терминах их характеристик, которые определяются так же, как и в случае эволюционных систем на евклидовом пространстве.

В дальнейшем предполагается, что все линейные операции выполняются над $\mathbb{R}$, и все бесконечномерные линейные пространства снабжены естественной локально выпуклой топологией. Все вводимые ниже линейные отображения непрерывные, хотя в явном виде это не доказывается. Используются обычные обозначения: $\mathbb{N}=$ $\{1,2, \ldots\}, \mathbb{Z}_{+}=\{0,1,2, \ldots\}, \mathbb{Z}=\{0, \pm 1, \pm 2, \ldots\}$. Через $\operatorname{Map}(X ; Y)$ обозначается множество всех отображений из множества $X$ в множество $Y$.

\section{1. РАССЛОЕНИЕ ДЖЕТОВ НАД РЕШЕТКОЙ}

1.1. Решетка. Пусть $D \in \mathbb{N}$. Решетка (точнее, $D$-мерная решетка) есть множество $\mathbb{L}=\mathbb{Z}^{D}$. На решетке $\mathbb{L}$ определены две алгебраические структуры: абелевой группы по сложению и $\mathbb{Z}$-модуля. Как $\mathbb{Z}$-модуль $\mathbb{L}$ имеет базис $\left\{e_{1}, \ldots, e_{D}\right\} \subset \mathbb{L}$, где $e_{1}=(1,0, \ldots, 0), \ldots, e_{D}=(0, \ldots, 0,1)$, так что

$$
\mathbb{L} \ni m=\left(\mu^{1}, \ldots, \mu^{D}\right)=\sum_{\alpha} \mu^{\alpha} e_{\alpha}, \quad \mu^{\alpha} \in \mathbb{Z}, \quad 1 \leqslant \alpha \leqslant D .
$$

* Математический институт им. В. А. Стеклова РАН, Москва, Россия. E-mail: zharinov@mi.ras.ru 
Для $m=\left(\mu^{1}, \ldots, \mu^{D}\right) \in \mathbb{L}$ положим $|m|=\sum_{\alpha}\left|\mu^{\alpha}\right| \in \mathbb{Z}_{+}$. Для краткости будем писать $\{|m| \leqslant p\}$ вместо $\{m \in \mathbb{L} ;|m| \leqslant p\}$, где $p \in \mathbb{Z}_{+}$.

На решетке $\mathbb{L}$ определено действие группы $\mathbb{L}$,

$$
\mathbb{L} \ni n \mapsto T_{n} \in \operatorname{Map}(\mathbb{L} ; \mathbb{L}), \quad m \mapsto T_{n} m=m+n ;
$$

отображения $T_{n}$ называются сдвигами.

1.2. Векторное расслоение. Пусть $V$ - конечномерное линейное пространство. Будем рассматривать прямое произведение $E=\mathbb{L} \times V$ как векторное расслоение над решеткой $\mathbb{L}$ с типичным слоем $V$ и проекцией на первый сомножитель:

$$
\pi: \mathbb{L} \times V \rightarrow \mathbb{L}, \quad(m, u) \mapsto \pi(m, u)=m .
$$

Хотя с точки зрения классической дифференциальной геометрии это расслоение тривиальное, такой подход помогает адекватно переносить геометрические конструкции на дискретный случай. Сечения расслоения $\pi$ суть отображения из $\mathbb{L}$ в $V$. Линейное пространство всех таких отображений обозначим через $\operatorname{Map}(\mathbb{L} ; V)$. Носитель отображения $\phi \in \operatorname{Map}(\mathbb{L} ; V)$ есть множество $\operatorname{supp} \phi=\{m \in \mathbb{L} ; \phi(m) \neq 0\}$. Пусть $\operatorname{Map}_{0}(\mathbb{L} ; V)$ есть множество всех $\phi \in \operatorname{Map}(\mathbb{L} ; V)$ с конечными носителями.

Считая, что сдвиги действуют на слоях тривиально, получаем действие группы $\mathbb{L}$ на расслоении $\pi$ :

$$
\mathbb{L} \times V \ni(m, u) \mapsto T_{n}(m, u)=\left(T_{n} m, u\right) \in \mathbb{L} \times V \quad \text { для всех } \quad m, n \in \mathbb{L} .
$$

На сечениях имеем $\operatorname{id}_{V} \circ T_{n} \phi=\phi \circ T_{n}$, т.е. $T_{n} \phi=\phi \circ T_{n}$, так что

$$
\left(T_{n} \phi\right)(m)=\phi(m+n) \quad \text { для всех } \quad \phi \in \operatorname{Map}(\mathbb{L} ; V), \quad m, n \in \mathbb{L} .
$$

1.3. Расслоение джетов. Пусть $\pi: E=\mathbb{L} \times V \rightarrow \mathbb{L}-$ векторное расслоение над $\mathbb{L}$. Будем говорить, что сечения $\phi, \psi \in \operatorname{Map}(\mathbb{L} ; V)$ касаются над точкой $m \in \mathbb{L}$ $c$ порядком $p \in \mathbb{Z}_{+}$, если $\phi(m+n)=\psi(m+n)$ для всех $|n| \leqslant p$. Будем также говорить, что сечения $\phi, \psi \in \operatorname{Map}(\mathbb{L} ; V)$ касаются над точкой $m \in \mathbb{L}$ с порядком $\infty$, если $\phi(m+n)=\psi(m+n)$ для всех $n \in \mathbb{L}$, т.е. если сечения $\phi$ и $\psi$ совпадают.

При таком определении касания векторное расслоение джетов порядка $p \in \mathbb{Z}_{+}$ над $\mathbb{L}$ есть

$$
\pi^{p}: \mathcal{J}^{p}(\pi)=\mathbb{L} \times \mathbf{V}^{p} \rightarrow \mathbb{L}
$$

где типичный слой

$$
\mathbf{V}^{p}=\left\{\mathbf{u}^{p}=\left(u_{k} \in V ;|k| \leqslant p\right)\right\},
$$

$\pi^{p}$ - проекция на первый сомножитель, $\left(m, \mathbf{u}^{p}\right) \mapsto \pi^{p}\left(m, \mathbf{u}^{p}\right)=m$. Можно считать, что $\mathbf{V}^{0}=V, \mathcal{J}^{0}(\pi)=E, \pi^{0}=\pi$. Сечения расслоения $\pi^{p}$ суть отображения из $\mathbb{L}$ в $\mathbf{V}^{p}$. Линейное пространство всех таких отображений обозначим через

$$
\operatorname{Map}\left(\mathbb{L} ; \mathbf{V}^{p}\right)=\left\{\phi^{p}=\left(\phi_{k} \in \operatorname{Map}(\mathbb{L} ; V) ;|k| \leqslant p\right)\right\}
$$

(иначе, $\left(\phi^{p}\right)_{k}=\phi_{k}$ и $\left(\phi^{p}\right)_{k}(m)=\phi_{k}(m)$ для всех $\left.|k| \leqslant p, m \in \mathbb{L}\right)$. Каждому сечению $\phi \in \operatorname{Map}(\mathbb{L} ; V)$ ставится в соответствие его $p$-джет $\mathbf{j}^{p}(\phi) \in \operatorname{Map}\left(\mathbb{L} ; \mathbf{V}^{p}\right)$, где 
$\mathbf{j}^{p}(\phi)=\left(\phi_{k}=T_{k} \phi \in \operatorname{Map}(\mathbb{L} ; V) ;|k| \leqslant p\right)$, так что $\left(\mathbf{j}^{p} \phi\right)(m)=\left(\phi_{k}(m)=\phi(m+k) ;\right.$ $|k| \leqslant p), m \in \mathbb{L}$ (коротко, $\left.\left(\mathbf{j}^{p} \phi\right)_{k}(m)=\phi(m+k)\right)$. По построению сечения $\phi, \psi$ касаются над точкой $m$ с порядком $p$ тогда и только тогда, когда $\phi^{p}(m)=\boldsymbol{\psi}^{p}(m)$, т.е. когда их $p$-джеты в точке $m$ совпадают. Правило $\phi \mapsto \mathbf{j}^{p}(\phi)$ определяет линейное инъективное отображение (вложение) $\mathbf{j}^{p}: \operatorname{Map}(\mathbb{L} ; V) \rightarrow \operatorname{Map}\left(\mathbb{L} ; \mathbf{V}^{p}\right)$.

Пусть $p, q \in \mathbb{Z}_{+}, p \geqslant q$. Естественное вложение $\{|k| \leqslant q\} \subset\{|k| \leqslant p\}$ определяет проекцию

$$
\pi_{q}^{p}: \mathcal{J}^{p}(\pi) \rightarrow \mathcal{J}^{q}(\pi), \quad \pi^{q} \circ \pi_{q}^{p}=\pi^{p},
$$

где $\left(m, \mathbf{u}^{p}\right) \mapsto \pi_{q}^{p}\left(m, \mathbf{u}^{p}\right)=\left(m, \mathbf{u}^{q}\right), \mathbf{u}^{q}=\left(u_{k} \in \mathbf{u}^{p} ;|k| \leqslant q\right)$. Очевидно, $\pi_{r}^{q} \circ \pi_{q}^{p}=\pi_{r}^{p}$ при $p \geqslant q \geqslant r$, так что определен проективный предел - векторное расслоение джетов бесконечного порядка

$$
\pi^{\infty}: \mathcal{J}^{\infty}(\pi)=\mathbb{L} \times \mathbf{V} \rightarrow \mathbb{L}
$$

где типичный слой

$$
\mathbf{V}=\mathbf{V}^{\infty}=\operatorname{limproj}_{p \rightarrow \infty} \mathbf{V}^{p}=\left\{\mathbf{u}=\left(u_{k} \in V ; k \in \mathbb{L}\right)\right\},
$$

$\pi^{\infty}$ - проекция на первый сомножитель, $(m, \mathbf{u}) \mapsto \pi^{\infty}(m, \mathbf{u})=m$. Также определены проекции $\pi_{p}^{\infty}: \mathcal{J}^{\infty}(\pi) \rightarrow \mathcal{J}^{p}(\pi), p \in \mathbb{Z}_{+}$, причем $\pi^{p} \circ \pi_{p}^{\infty}=\pi^{\infty}$ и $\pi_{q}^{p} \circ \pi_{p}^{\infty}=\pi_{q}^{\infty}$ при $p \geqslant q$. Сечения расслоения $\pi^{\infty}$ суть отображения из $\mathbb{L} \mathrm{B} \mathbf{V}$, линейное пространство всех таких отображений есть

$$
\operatorname{Map}(\mathbb{L} ; \mathbf{V})=\left\{\boldsymbol{\phi}=\left(\phi_{k} \in \operatorname{Map}(\mathbb{L} ; V) ; k \in \mathbb{L}\right)\right\}
$$

(т.е. $(\phi)_{k}(m)=(\phi(m))_{k}=\phi_{k}(m)$ для всех $\left.k, m \in \mathbb{L}\right)$. Каждому сечению $\phi \in$ $\operatorname{Map}(\mathbb{L} ; V)$ ставится в соответствие его $\infty$-джет $\mathbf{j}(\phi) \in \operatorname{Map}(\mathbb{L} ; \mathbf{V})$, где $\mathbf{j}(\phi)=\left(\phi_{k}=\right.$ $\left.T_{k} \phi \in \operatorname{Map}(\mathbb{L} ; V) ; k \in \mathbb{L}\right),(\mathbf{j} \phi)_{k}(m)=\phi(m+k), m, k \in \mathbb{L}$. По построению сечения $\phi, \psi$ касаются над точкой $m$ с порядком $\infty$ тогда и только тогда, когда $\phi(m)=\boldsymbol{\psi}(m)$, т.е. когда их $\infty$-джеты в точке $m$ совпадают. Вложение $\mathbf{j}: \operatorname{Map}(\mathbb{L} ; V) \rightarrow \operatorname{Map}(\mathbb{L} ; \mathbf{V})$ задается правилом $\phi \mapsto \mathbf{j}(\phi)$, причем $\mathbf{j}^{p}=\pi_{p}^{\infty} \circ \mathbf{j}, p \in \mathbb{Z}_{+}$.

По построению $\mathbf{V} \simeq \operatorname{Map}(\mathbb{L} ; V)$. По аналогии с теорией гладких джетов определим действие группы $\mathbb{L}$ на типичном слое $\mathbf{V}$ согласно ее действию на $\operatorname{Map}(\mathbb{L} ; V)$. А именно, для всех $n \in \mathbb{L}$ положим

$$
T_{n}: \mathbf{V} \rightarrow \mathbf{V}, \quad \mathbf{u} \rightarrow \mathbf{v}=T_{n} \mathbf{u}, \quad\left(T_{n} \mathbf{u}\right)_{k}=u_{T_{n} k}=u_{k+n} \quad \text { для всех } \quad k \in \mathbb{L} .
$$

$\mathrm{B}$ частности, получаем действие группы $\mathbb{L}$ на расслоении $\pi^{\infty}$ :

$$
\mathbb{L} \times \mathbf{V} \ni(m, \mathbf{u}) \mapsto T_{n}(m, \mathbf{u})=\left(T_{n} m, T_{n} \mathbf{u}\right) \in \mathbb{L} \times \mathbf{V}, \quad n \in \mathbb{L}
$$

На сечениях соответственно имеем $T_{n} \circ T_{n} \phi=\phi \circ T_{n}$, откуда

$$
T_{n} \phi=T_{-n} \circ \phi \circ T_{n} \quad \text { для всех } \quad n \in \mathbb{L}, \quad \phi \in \operatorname{Map}(\mathbb{L} ; \mathbf{V}),
$$

т.e.

$$
\left(T_{n} \phi\right)_{k}(m)=\phi_{k-n}(m+n) \quad \text { для всех } \quad \phi=\left(\phi_{k} \in \operatorname{Map}(\mathbb{L} ; V)\right), \quad k, m, n \in \mathbb{L} .
$$


ПрЕДЛОЖЕНИЕ 1. Имеют место следующие утверждения.

1. $T_{n} \circ \mathbf{j}=\mathbf{j}$ для всех $n \in \mathbb{L}$.

2. Сечение $\phi \in \operatorname{Map}(\mathbb{L} ; \mathbf{V})$ является $\infty$-джетом некоторого сечения $\phi \in$ $\operatorname{Map}(\mathbb{L} ; V), \phi=\mathbf{j}(\phi)$, тогда и только тогда, когда оно инвариантно относительно сдвигов, т.е. когда $T_{n} \phi=\phi$ для всех $n \in \mathbb{L}$.

3. Для любой точки $(m, \mathbf{u}) \in \mathcal{J}^{\infty}(\pi)$ существует единственное сечение $\phi \in$ $\operatorname{Map}(\mathbb{L} ; V)$ maкое, что $(\mathbf{j} \phi)(m)=\mathbf{u}$.

\section{2. ОПЕРАЦИИ НА РАССЛОЕНИИ РАЗНОСТНЫХ ДЖЕТОВ}

2.1. Функции на разностных джетах. Пусть $\pi: \mathbb{L} \times V \rightarrow \mathbb{L}-$ векторное расслоение, $\mathcal{J}^{p}(\pi)$ - расслоение джетов порядка $p \in \mathbb{Z}_{+}, W$ - линейное пространство. Будем рассматривать прямое произведение $\mathcal{J}^{p}(\pi) \times W$ как векторное расслоение над $\mathcal{J}^{p}(\pi)$ с типичным слоем $W$ и проекцией на первый сомножитель

$$
\mathcal{J}^{p}(\pi) \times W \rightarrow \mathcal{J}^{p}(\pi), \quad\left(m, \mathbf{u}^{p}, w\right) \mapsto\left(m, \mathbf{u}^{p}\right) .
$$

Сечения этого расслоения суть отображения из $\mathcal{J}^{p}(\pi)=\mathbb{L} \times \mathbf{V}^{p}$ в $W$, линейное пространство всех таких отображений есть $\operatorname{Map}\left(\mathbb{L} \times \mathbf{V}^{p} ; W\right)$. Отображение $f\left(m, \mathbf{u}^{p}\right) \in$ $\operatorname{Map}\left(\mathbb{L} \times \mathbf{V}^{p} ; W\right)$ назовем гладким, если $f_{m}\left(\mathbf{u}^{p}\right)=f\left(m, \mathbf{u}^{p}\right) \in \operatorname{Map}\left(\mathbf{V}^{p} ; W\right)$ - гладкое отображение (т.е. принадлежит классу $\mathcal{C}^{\infty}$ ) по $\mathbf{u}^{p} \in \mathbf{V}^{p}$ при каждом $m \in \mathbb{L}$. Линейное пространство всех таких гладких отображений обозначим через $\mathcal{M}\left(\mathbb{L} \times \mathbf{V}^{p}, W\right)$. По построению $\mathcal{M}\left(\mathbb{L} \times \mathbf{V}^{p}, W\right)=\operatorname{Map}\left(\mathbb{L} ; \mathcal{C}^{\infty}\left(\mathbf{V}^{p} ; W\right)\right)$. При $p, q \in \mathbb{Z}_{+}, p \leqslant q$, имеются естественные вложения $\mathcal{M}\left(\mathbb{L} \times \mathbf{V}^{p}, W\right) \subset \mathcal{M}\left(\mathbb{L} \times \mathbf{V}^{q}, W\right)$, порожденные проекциями $\pi_{p}^{q}: \mathcal{J}^{q}(\pi) \rightarrow \mathcal{J}^{p}(\pi)$. Таким образом, определен индуктивный предел линейных пространств

$$
\begin{aligned}
\mathcal{M}(\mathbb{L} \times \mathbf{V}, W) & =\operatorname{limind}_{p \rightarrow \infty} \mathcal{M}\left(\mathbb{L} \times \mathbf{V}^{p}, W\right)= \\
& =\operatorname{limind}_{p \rightarrow \infty} \operatorname{Map}\left(\mathbb{L} ; \mathcal{C}^{\infty}\left(\mathbf{V}^{p} ; W\right)\right)=\operatorname{Map}\left(\mathbb{L} ; \mathcal{C}^{\infty}(\mathbf{V} ; W)\right) .
\end{aligned}
$$

По построению отображение $f=f(m, \mathbf{u}) \in \mathcal{M}(\mathbb{L} \times \mathbf{V}, W)$, если существует число $p=p(f) \in \mathbb{Z}_{+}(p-$ порядок $f)$ такое, что $f \in \mathcal{M}\left(\mathbb{L} \times \mathbf{V}^{p}, W\right)$, но $f \notin \mathcal{M}\left(\mathbb{L} \times \mathbf{V}^{p-1}, W\right)$.

Таким образом, определены пространства:

1) $\mathcal{M}=\mathcal{M}(\mathbb{L} \times \mathbf{V} ; W)=\operatorname{Map}\left(\mathbb{L} ; \mathcal{C}^{\infty}(\mathbf{V} ; W)\right)$;

2) $\mathcal{A}=\mathcal{A}(\mathbb{L} \times \mathbf{V})=\mathcal{M}(\mathbb{L} \times \mathbf{V} ; \mathbb{R})=\operatorname{Map}\left(\mathbb{L} ; \mathcal{C}^{\infty}(\mathbf{V})\right)$ (здесь $\left.W=\mathbb{R}\right)$;

3) $\mathcal{E}=\mathcal{M}(\mathbb{L} \times \mathbf{V} ; V)=\operatorname{Map}\left(\mathbb{L} ; \mathcal{C}^{\infty}(\mathbf{V} ; V)\right)$ (здесь $\left.W=V\right)$;

4) $\mathcal{E}^{*}=\mathcal{M}\left(\mathbb{L} \times \mathbf{V} ; V^{*}\right)=\operatorname{Map}\left(\mathbb{L} ; \mathcal{C}^{\infty}\left(\mathbf{V} ; V^{*}\right)\right.$ ) (здесь $W=V^{*}$ - дуальное пространство).

Заметим, что $\mathcal{A}$ есть алгебра функций с обычными поточечными операциями, а $\mathcal{M}$, $\mathcal{E}$ и $\mathcal{E}^{*}$ суть $\mathcal{A}$-модули.

Ради простоты обозначений для данных функций $f=f(m, \mathbf{u}) \in \mathcal{M}, \boldsymbol{\phi}=\boldsymbol{\phi}(m) \in$ $\operatorname{Map}(\mathbb{L} ; \mathbf{V})$ будем писать

$$
\left.f\right|_{\phi}=\left.f\right|_{\phi}(m)=f(m, \phi(m)) \in \operatorname{Map}(\mathbb{L} ; W) .
$$


Отображения $f \in \mathcal{M}$ суть сечения векторного расслоения

$$
\mathcal{J}^{\infty}(\pi) \times W \rightarrow \mathcal{J}^{\infty}(\pi), \quad(m, \mathbf{u}, w) \mapsto(m, \mathbf{u}) .
$$

Считая, что сдвиги действуют на слоях тривиально, получаем действие группы $\mathbb{L}$ на этом расслоении:

$$
\mathcal{J}^{\infty}(\pi) \times W \ni(m, \mathbf{u}, w) \mapsto T_{n}(m, \mathbf{u}, w)=\left(T_{n} m, T_{n} \mathbf{u}, w\right) \in \mathcal{J}^{\infty}(\pi) \times W
$$

для всех $n \in \mathbb{L}$. На сечениях соответственно имеем $\operatorname{id}_{W} \circ T_{n} f=f \circ T_{n}$, откуда

$$
\left(T_{n} f\right)(m, \mathbf{u})=f\left(T_{n} m, T_{n} \mathbf{u}\right) \quad \text { для всех } f \in \mathcal{M}, \quad m, n \in \mathbb{L}, \quad \mathbf{u} \in \mathbf{V} .
$$

ПреДлОжЕНИЕ 2 (правило полного сдвига). Для данной функции $\phi=\left(\phi_{k}\right) \in$ $\operatorname{Map}(\mathbb{L} ; \mathbf{V})$ равенство

$$
\left.\left(T_{n} f\right)\right|_{\phi}=T_{n}\left(\left.f\right|_{\phi}\right) \quad \text { для всех } \quad n \in \mathbb{L}, \quad f \in \mathcal{M}
$$

справедливо тогда и только тогда, когда $\boldsymbol{\phi}=\mathbf{j}(\phi)$, где $\phi=\phi_{0}$.

ДоказАтельство. По определению $\left(T_{n} f\right)(m, \phi(m))=f\left(T_{n} m, T_{n}(\phi(m))\right)$, так что надо доказать, что $T_{n}(\phi(m))=\phi\left(T_{n} m\right)$ для всех $m, n \in \mathbb{L}$ тогда и только тогда, когда $\phi_{k}=T_{k} \phi_{0}$ для всех $k \in \mathbb{L}$. Но, с одной стороны,

$$
\left(T_{n}(\phi(m))\right)_{k}=(\phi(m))_{k+n}=\phi_{k+n}(m),
$$

а с другой стороны,

$$
\left(\phi\left(T_{n} m\right)\right)_{k}=\phi_{k}\left(T_{n} m\right)=\phi_{k}(m+n) .
$$

Следовательно, нужное равенство имеет место тогда и только тогда, когда $\phi_{k+n}(m)$ $=\phi_{k}(m+n)$ для всех $k, m, n \in \mathbb{L}$, или, эквивалентно (при $\left.k=0\right)$, когда $\phi_{n}(m)=$ $\phi_{0}(m+n)=\left(T_{n} \phi_{0}\right)(m)$ для всех $m, n \in \mathbb{L}$, т.е. когда $\phi_{n}=T_{n} \phi_{0}$ для всех $n \in \mathbb{L}$, что и требовалось доказать.

2.2. Вертикальные дифференцирования. Пусть $\mathfrak{D}_{V}-$ множество всех дифференцирований алгебры $\mathcal{A}$ и модуля $\mathcal{M}$. Как известно, $\mathfrak{D}_{V}$ обладает структурой алгебры Ли с коммутатором в качестве скобки Ли и структурой $\mathcal{A}$-модуля с операцией

$$
\mathcal{A} \times \mathfrak{D}_{V} \ni(f, X) \mapsto Y=f X \in \mathfrak{D}_{V}, \quad(f X) g=f(X g), \quad g \in \mathcal{A} \quad \text { или } \quad \mathcal{M} .
$$

ЗАмЕчАниЕ 1. Обратим внимание, что дифференцирования производятся по непрерывным переменным $\mathbf{u}$, а дискретные переменные $m$ здесь играют роль параметров. Учитывая этот факт, дифференцирования в алгебре $\mathcal{A}$ и модуле $\mathcal{M}$ будем называть вертикальными. В свою очередь, сдвиги в $\mathcal{A}$ и $\mathcal{M}$ являются подъемом сдвигов из базы $\mathbb{L}$ и в силу этого называются горизонтальными. По определению вертикальные дифференцирования $X \in \mathfrak{D}_{V}$ удовлетворяют правилу Лейбница, $X(f g)=(X f) g+f(X g)$ для всех $f \in \mathcal{A}, g \in \mathcal{A}$ или $\mathcal{M}$, а горизонтальные сдвиги являются морфизмами алгебры $\mathcal{A}$ и модуля $\mathcal{M}$, т.е. $T_{n}(f g)=\left(T_{n} f\right)\left(T_{n} g\right)$ для всех $n \in \mathbb{L}, f \in \mathcal{A}$ и $g \in \mathcal{A}$ или $\mathcal{M}$. Соответственно вертикальные дифференцирования образуют алгебру Ли, а горизонтальные сдвиги - абелеву группу. 
Пусть $\left\{a_{i} ; i \in I\right\}$ - базис конечномерного линейного пространства $V$ :

$$
V \ni u=\left(u^{i}\right)=\sum_{i} u^{i} a_{i}, \quad u^{i} \in \mathbb{R}, \quad i \in I .
$$

Тогда дуальное пространство $V^{*}$ имеет дуальный базис $\left\{b^{i} ; i \in I\right\},\left\langle b^{i}, a_{j}\right\rangle=\delta_{j}^{i}$ для всех $i, j \in I$, и

$$
V^{*} \ni \omega=\left(\omega_{i}\right)=\sum_{i} \omega_{i} b^{i}, \quad \omega \in \mathbb{R}, \quad i \in I .
$$

Также имеем представление

$$
\mathbf{V} \ni \mathbf{u}=\left(u_{m}^{i}\right), \quad u_{m}^{i} \in \mathbb{R}, \quad i \in I, \quad m \in \mathbb{L} .
$$

Модуль $\mathfrak{D}_{V}$ имеет в этом случае базис $\left\{\partial_{u_{m}^{i}} ; i \in I, m \in \mathbb{L}\right\}$, где $\partial_{u_{m}^{i}}-$ частная производная по переменной $u_{m}^{i}$, так что

$$
\mathfrak{D}_{V} \ni X=\left(X_{m}^{i}\right)=\sum_{i, m} X_{m}^{i} \partial_{u_{m}^{i}}, \quad X_{m}^{i} \in \mathcal{A}, \quad i \in I, \quad m \in \mathbb{L} .
$$

Далее, дуальный модуль $\mathfrak{D}_{V}^{*}=\operatorname{Hom}_{\mathcal{A}}\left(\mathfrak{D}_{V} ; \mathcal{A}\right)$ имеет дуальный базис $\left\{\vartheta_{m}^{i} ; i \in I\right.$, $m \in \mathbb{L}\},\left\langle\vartheta_{m}^{i}, \partial_{u_{n}^{j}}\right\rangle=\vartheta_{m}^{i}\left(\partial_{u_{n}^{j}}\right)=\delta_{j}^{i} \delta_{m}^{n}, i, j \in I, m, n \in \mathbb{L}$. Тем самым

$$
\mathfrak{D}_{V}^{*} \ni \omega=\left(\omega_{i}^{m}\right)=\sum_{i, m} \omega_{i}^{m} \vartheta_{m}^{i}, \quad \omega_{i}^{m} \in \mathcal{A}, \quad i \in I, \quad m \in \mathbb{L} .
$$

Пусть $X \in \mathfrak{D}_{V}$. Действие $X: \mathfrak{D}_{V} \rightarrow \mathfrak{D}_{V}$ определяется как присоединенное, $X(Y)=$ $[X, Y]$ для всех $Y \in \mathfrak{D}_{V}$. Дуальным образом действие $X: \mathfrak{D}_{V}^{*} \rightarrow \mathfrak{D}_{V}^{*}$ определяется как производная Ли, т.е. условием

$$
X(\langle\omega, Y\rangle)=\langle X(\omega), Y\rangle+\langle\omega, X(Y)\rangle, \quad \omega \in \mathfrak{D}_{V}^{*}, \quad Y \in \mathfrak{D}_{V},
$$

т.е. $X(\omega)=[X, \omega]$ для всех $\omega \in \mathfrak{D}_{V}^{*}$. В частности, $\partial_{u_{m}^{i}}\left(\vartheta_{n}^{j}\right)=0, i, j \in I, m, n \in \mathbb{L}$.

Пусть $n \in \mathbb{L}$. Действие $T_{n}: \mathfrak{D}_{V} \rightarrow \mathfrak{D}_{V}$ определяется как подъем действия с $\mathcal{A}$, т.е. условием

$$
X \mapsto T_{n}(X), \quad T_{n}(X) \circ T_{n}=T_{n} \circ X, \quad X \in \mathfrak{D}_{V},
$$

в частности, $T_{n}\left(\partial_{u_{m}^{i}}\right)=\partial_{u_{m+n}^{i}}, i \in I, m \in \mathbb{L}$. Дуальным образом действие $T_{n}: \mathfrak{D}_{V}^{*} \rightarrow$ $\mathfrak{D}_{V}^{*}$ определяется правилом $T_{n}\langle\omega, X\rangle=\left\langle T_{n} \omega, T_{n} X\right\rangle$, т.е.

$$
\omega \mapsto T_{n} \omega, \quad T_{n}(\omega) \circ T_{n}=T_{n} \circ \omega, \quad \omega \in \mathfrak{D}_{V}^{*} .
$$

В частности, $T_{n}\left(\vartheta_{m}^{i}\right)=\vartheta_{m+n}^{i}, i \in I, m \in \mathbb{L}$.

ПреДЛОЖЕНИЕ 3. Пусть $X \in \mathfrak{D}_{V}$. Равенство $T_{n}(X)=X$ (другими словами, равенство $\left.\left[X, T_{n}\right]=0\right)$ справедливо для всех $n \in \mathbb{L}$ тогда и только тогда, когда

$$
X=\sum_{i, m}\left(T_{m} f^{i}\right) \partial_{u_{m}^{i}}=\mathrm{ev}_{f}, \quad f=\left(f^{i}\right) \in \mathcal{E},
$$

есть эволюиионное дифференцирование. 
Доказательство. Действительно, с одной стороны, для всех $f \in \mathcal{E}$ и всех $n \in \mathbb{L}$ имеем

$$
T_{n} \circ \operatorname{ev}_{f}=\sum_{i, m}\left(T_{n+m} f^{i}\right)\left(T_{n} \circ \partial_{u_{m}^{i}}\right)=\sum_{i, m}\left(T_{m+n} f^{i}\right)\left(\partial_{u_{m+n}^{i}} \circ T_{n}\right)=\operatorname{ev}_{f} \circ T_{n}
$$

С другой стороны, пусть $X=\sum_{i, m} X_{m}^{i} \partial_{u_{m}^{i}} \in \mathfrak{D}_{V}$ и $X=T_{n} \circ X \circ T_{-n}$ для всех $n \in \mathbb{L}$, т.е.

$$
\begin{aligned}
X & =\sum_{i, m} X_{m}^{i} \partial_{u_{m}^{i}}=\sum_{i, m}\left(T_{n} X_{m}^{i}\right)\left(T_{n} \circ \partial_{u_{m}^{i}} \circ T_{-n}\right)=\sum_{i, m}\left(T_{n} X_{m}^{i}\right) \partial_{u_{m+n}^{i}}= \\
& =\sum_{i, m}\left(T_{n} X_{m-n}^{i}\right) \partial_{u_{m}^{i}}
\end{aligned}
$$

Следовательно, $X_{m}^{i}=T_{n} X_{m-n}^{i}$ для всех $i \in I, n, m \in \mathbb{L}$. Полагая $n=m$, получаем $X_{m}^{i}=T_{m} X_{0}^{i}$ для всех $i \in I, m \in \mathbb{L}$, откуда $X=\operatorname{ev}_{f}$, где $f=X_{0} \in \mathcal{E}$.

Легко проверяется, что коммутатор $\left[\mathrm{ev}_{f}, \mathrm{ev}_{g}\right]=\mathrm{ev}_{h}$ для всех $f, g \in \mathcal{E}$, где $h=$ $\left(h^{i}\right) \in \mathcal{E}, h^{i}=\mathrm{ev}_{f}\left(g^{i}\right)-\mathrm{ev}_{g}\left(f^{i}\right), i \in I$. Следовательно, определена алгебра Ли эволюиионных дифференцирований $\mathfrak{D}_{E}=\left\{\mathrm{ev}_{f} ; f \in \mathcal{E}\right\}-$ подалгебра алгебры Ли $\mathfrak{D}_{V}$.

2.3. Горизонтальные разностные операторы. Пусть $1 \leqslant \alpha \leqslant D$. Разностные операторы $\Delta_{\alpha}=T_{e_{\alpha}}-T_{0}$ действуют во всех пространствах, где определены сдвиги. Введем линейное пространство $\mathfrak{D}_{H}=\left\{\zeta=\sum_{\alpha} \zeta^{\alpha} \Delta_{\alpha} ; \zeta^{\alpha} \in \mathbb{R}\right\} \simeq \mathbb{R}^{D}$ и рассмотрим его как коммутативную алгебру Ли, порожденную операторами $\Delta_{1}, \ldots, \Delta_{D}$. Ее элементы действуют во всех пространствах, где определены сдвиги. Дуальное пространство $\mathfrak{D}_{H}^{*}=\operatorname{Hom}_{\mathbb{R}}\left(\mathfrak{D}_{H} ; \mathbb{R}\right)$ имеет дуальный базис $\left\{\Theta^{1}, \ldots, \Theta^{D}\right\},\left\langle\Theta^{\alpha}, \Delta_{\beta}\right\rangle=$ $\Theta^{\alpha}\left(\Delta_{\beta}\right)=\delta_{\beta}^{\alpha}$. Поскольку группа $\mathbb{L}$ коммутативна, она действует на $\mathfrak{D}_{H}$ и $\mathfrak{D}_{H}^{*}$ тривиально.

2.4. Вариационное исчисление на решетке. Будем говорить, что функция $f \in \operatorname{Map}(\mathbb{L} ; W)$ суммируемая, если ряд $\sum f=\sum_{m \in \mathbb{L}} f(m)$ сходится в топологии пространства $W$.

ПреДЛОЖЕНИЕ 4. Пусть функиия $f \in \operatorname{Map}(\mathbb{L} ; W)$ суммируемая. Тогда функиия $T_{n} f \in \operatorname{Map}(\mathbb{L} ; W)$ также суммируемая для любого $n \in \mathbb{L}$, причем $\sum T_{n} f=\sum f$.

ДоказАтельство. Действительно, в этом случае $\sum_{m} f(m+n)=\sum_{k} f(k)$.

Следуя традиции, будем называть функции $\lambda \in \mathcal{A}$ лагранжсианами.

Каждый лагранжиан $\lambda \in \mathcal{A}$ порождает функционал (иными словами, действие) $\Lambda$ на $\operatorname{Map}(\mathbb{L} ; V)$ (точнее, $\Lambda \in \operatorname{Map}(\operatorname{Map}(\mathbb{L} ; V) ; \mathbb{R}))$ по правилу

$$
\Lambda(\phi)=\left.\sum_{m} \lambda\right|_{\mathbf{j}(\phi)}(m), \quad \phi \in \operatorname{Map}(\mathbb{L} ; \mathbb{R})
$$

ЗАмЕчАниЕ 2. Здесь неявно подразумевается, что область определения функционала $\Lambda$ есть множество всех $\phi \in \operatorname{Map}(\mathbb{L} ; V)$, для которых указанный ряд сходится. 
Каждый лагранжиан $\lambda \in \mathcal{A}$ индуцирует два линейных отображения:

1) $\lambda_{*}: \mathcal{E} \rightarrow \mathcal{A}, f=\left(f^{i}\right) \mapsto \lambda_{*}(f)=\operatorname{ev}_{f}(\lambda)=\sum_{i, n}\left(\partial_{u_{n}^{i}} \lambda\right) T_{n} f^{i}$

2) $\lambda^{*}: \mathcal{A} \rightarrow \mathcal{E}^{*}, \varrho \mapsto \lambda^{*}(\varrho)=\left(\lambda^{*}(\varrho)_{i}\right), \lambda^{*}(\varrho)_{i}=\sum_{n} T_{-n}\left(\varrho \partial_{u_{n}^{i}} \lambda\right)$.

ЗАмЕчАниЕ 3 . Обратим внимание, что частная производная $\partial_{u_{n}^{i}} \lambda \neq 0$ лишь для конечного числа $n \in \mathbb{L}$.

ПРЕДЛОЖЕНИЕ 5. Отображения $\lambda_{*} u \lambda^{*}$ сопряжены по Лагранжу:

$$
\left.\sum_{m}\left(\varrho \lambda_{*}(f)\right)\right|_{\mathbf{j}(\phi)}(m)=\left.\sum_{m}\left(\sum_{i} \lambda^{*}(\varrho)_{i} f^{i}\right)\right|_{\mathbf{j}(\phi)}(m)
$$

для всех $\lambda, \varrho \in \mathcal{A}, f \in \mathcal{E}, \phi \in \operatorname{Map}(\mathbb{L} ; V)$.

ДокАзАтЕльство. Действительно, с учетом предложений 2 и 4 имеем

$$
\begin{aligned}
& \left.\sum_{m}\left(\varrho \lambda_{*}(f)\right)\right|_{\mathbf{j}(\phi)}(m)=\left.\sum_{m, i, n}\left(\varrho\left(\partial_{u_{n}^{i}} \lambda\right) T_{n} f^{i}\right)\right|_{\mathbf{j}(\phi)}(m)= \\
& \quad=\left.\sum_{m, i, n}\left[T_{n}\left(T_{-n}\left(\varrho \partial_{u_{n}^{i}} \lambda\right) f^{i}\right)\right]\right|_{\mathbf{j}(\phi)}(m)=\sum_{i, n} \sum_{m}\left[T_{n}\left(\left.\left(T_{-n}\left(\varrho \partial_{u_{n}^{i}} \lambda\right) f^{i}\right)\right|_{\mathbf{j}(\phi)}\right)\right](m)= \\
& \quad=\left.\sum_{i, m, n}\left(\left(T_{-n}\left(\varrho \partial_{u_{n}^{i}} \lambda\right)\right) f^{i}\right)\right|_{\mathbf{j}(\phi)}(m)=\left.\sum_{m}\left(\sum_{i} \lambda^{*}(\varrho)_{i} f^{i}\right)\right|_{\mathbf{j}(\phi)}(m) .
\end{aligned}
$$

Вариация функционала $\Lambda$ определяется формулой

$$
\delta \Lambda(\phi ; \delta \phi)=\left.\sum_{m} \lambda_{*}(\delta \phi)\right|_{\mathbf{j}(\phi)}(m), \quad \phi \in \operatorname{Map}(\mathbb{L} ; V), \quad \delta \phi \in \operatorname{Map}_{0}(\mathbb{L} ; V) .
$$

ЗАмЕчАниЕ 4. Обратим внимание, что вариация $\delta \Lambda(\phi ; \delta \phi)$ определена для всех $\phi \in \operatorname{Map}(\mathbb{L} ; V)$ и $\delta \phi \in \operatorname{Map}_{0}(\mathbb{L} ; V)$.

ПреДЛОЖЕНИЕ 6. Имеет место представление

$$
\delta \Lambda(\phi ; \delta \phi)=\left.\sum_{m, i}\left(\delta_{u^{i}} \lambda\right)\right|_{\mathbf{j}(\phi)}(m) \delta \phi^{i}(m), \quad \phi \in \operatorname{Map}(\mathbb{L} ; V), \quad \delta \phi \in \operatorname{Map}_{0}(\mathbb{L} ; V),
$$

¿əe

$$
\delta_{u^{i}} \lambda=\frac{\delta \lambda}{\delta u^{i}}=\lambda^{*}(1)_{i}=\sum_{n} T_{-n}\left(\partial_{u_{n}^{i}} \lambda\right), \quad i \in I,
$$

- вариачионная производная (здесь $1 \in \mathcal{A})$.

Для доказательства достаточно воспользоваться предложением 5 .

В частности, определено линейное отображение

$$
\delta_{u}: \mathcal{A} \rightarrow \mathcal{E}^{*}, \quad \lambda \mapsto \delta_{u} \lambda, \quad\left(\delta_{u} \lambda\right)_{i}=\delta_{u^{i}} \lambda, \quad i \in I .
$$

Кроме того, имеется линейное отображение $\Delta \mathrm{iv}: \mathcal{A}^{D} \rightarrow \mathcal{A}(D$ - размерность решетки $\mathbb{L}$ ), которое каждому вектору $J=\left(J^{\alpha}\right) \in \mathcal{A}^{D}$ ставит в соответствие его разностную дивергениию

$$
\Delta \mathrm{iv} J=\sum_{\alpha} \Delta_{\alpha} J^{\alpha} \in \mathcal{A}
$$


ПрЕДЛОЖЕНИЕ 7. Вариация $\delta \Lambda(\phi ; \delta \phi)=0$ для всех $\phi \in \operatorname{Map}(\mathbb{L} ; V) u \delta \phi \in$ $\operatorname{Map}_{0}(\mathbb{L} ; V)$ тогда и только тогда, когда плотность $\lambda=\Delta \mathrm{iv} J$ для некоторого вектора $J \in \mathcal{A}^{D}$. В частности, $\operatorname{Im} \Delta \mathrm{iv}=\operatorname{Ker} \delta_{u}$, где $\operatorname{Im} \Delta \mathrm{iv}-$ образ отображения $\Delta \mathrm{iv}$, $a \operatorname{Ker} \delta_{u}-$ ядро отображения $\delta_{u}$.

ДокАЗАтЕльство. В соответствии с предложением 6 фактически следует доказать, что условие $\delta_{u} \lambda=0$ влечет представление $\lambda=\Delta \mathrm{iv} J$. Естественное доказательство последнего утверждения требует более глубокого развития разностного вариационного формализма, что выходит за рамки данной статьи. Этот факт считается известным (см., например, [7]-[9]), хотя автор и не нашел строгого доказательства.

ПреДЛОЖЕНИЕ 8. Для данного вектора $A \in \mathcal{A}^{D}$ равенство $\Delta \mathrm{iv} A=0$ имеет место тогда и толъко тогда, когда $A^{\alpha}=\sum_{\beta} \Delta_{\beta} B^{\alpha \beta}$, где компоненты $B^{\alpha \beta} \in \mathcal{A}$, $B^{\alpha \beta}+B^{\beta \alpha}=D, 1 \leqslant \alpha, \beta \leqslant 0$.

ДокАЗАтЕльство. Это утверждение является еще одной составляющей вариационного комплекса, широко известной в обычном вариационном исчислении (см., например, [10]). В разностной ситуации оно тоже считается известным (см., например, [8]), хотя строгое доказательство отсутствует. В любом случае доказательство точности разностного вариационного комплекса (и бикомплекса) - это тема отдельного исследования.

Каждая функция $\chi=\left(\chi_{i}\right) \in \mathcal{E}^{*}$ задает два линейных отображения:

1) $\chi_{*}: \mathcal{E} \rightarrow \mathcal{E}^{*}, f \mapsto \chi_{*}(f)$, где $\chi_{*}(f)_{i}=\sum_{j, n}\left(\partial_{u_{n}^{j}} \chi_{i}\right) T_{n} f^{j}$;

2) $\chi^{*}: \mathcal{E} \rightarrow \mathcal{E}^{*}, f \mapsto \chi^{*}(f)$, где $\chi^{*}(f)_{i}=\sum_{j, n}\left(\partial_{u^{i}} T_{n} \chi_{j}\right) T_{n} f^{j}$.

ПрЕДЛОЖЕНИЕ 9. Отображения $\chi_{*} u \chi^{*}$ сопряжены по Лагранжу:

$$
\left.\sum_{i, m}\left(g^{i} \chi_{*}(f)_{i}\right)\right|_{\mathbf{j}(\phi)}(m)=\left.\sum_{j, m}\left(\chi^{*}(g)_{j} f^{j}\right)\right|_{\mathbf{j}(\phi)}(m)
$$

для всех $f, g \in \mathcal{E}, \phi \in \operatorname{Map}(\mathbb{L} ; V)$.

ДокАЗАтЕЛьство. Действительно,

$$
\begin{aligned}
\left.\sum_{i, m}\left(g^{i} \chi_{*}(f)_{i}\right)\right|_{\mathbf{j}(\phi)}(m)=\left.\sum_{i, j, m, n}\left(g^{i}\left(\partial_{u_{n}^{j}} \chi_{i}\right) T_{n} f^{j}\right)\right|_{\mathbf{j}(\phi)}(m)= \\
\quad=\left.\sum_{i, j, n} \sum_{m}\left[T_{n}\left(T_{-n}\left(g^{i} \partial_{u_{n}^{j}} \chi_{i}\right) f^{j}\right)\right]\right|_{\mathbf{j}(\phi)}(m)=\left.\sum_{i, j, m, n}\left(T_{-n}\left(g^{i} \partial_{u_{n}^{j}} \chi_{i}\right) f^{j}\right)\right|_{\mathbf{j}(\phi)}(m)= \\
=\left.\sum_{i, j, m, n}\left(T_{n}\left(g^{i} \partial_{u_{-n}^{j}} \chi_{i}\right) f^{j}\right)\right|_{\mathbf{j}(\phi)}(m)=\left.\sum_{i, j, m, n}\left(\left(T_{n} g^{i}\right)\left(\partial_{u^{j}} T_{n} \chi_{i}\right) f^{j}\right)\right|_{\mathbf{j}(\phi)}(m)= \\
=\left.\sum_{j, m}\left(\chi^{*}(g)_{j} f^{j}\right)\right|_{\mathbf{j}(\phi)}(m) .
\end{aligned}
$$

ПредлОЖЕНИЕ 10. Функиия $\chi=\left(\chi_{i}\right) \in \mathcal{E}^{*}$ обладает представлением $\chi=\delta_{u} \lambda$ для некоторого $\lambda \in \mathcal{A}$ тогда и только тогда, когда $\chi_{*}=\chi^{*}$. Другими словами, образ $\operatorname{Im} \delta_{u}=\left\{\chi \in \mathcal{E}^{*} ; \chi_{*}=\chi^{*}\right\}$. 
ДокАЗАТЕЛьСтво. Действительно, с одной стороны, легко проверяется, что $\left(\delta_{u} \lambda\right)_{*}=\left(\delta_{u} \lambda\right)^{*}$ для всякого $\lambda \in \mathcal{A}$. С другой стороны, пусть $\chi \in \mathcal{E}^{*}$ и $\chi_{*}=\chi^{*}$, т.е. $\partial_{u_{n}^{j}} \chi_{i}=\partial_{u^{i}} T_{n} \chi_{j}$ для всех $i, j \in I, n \in \mathbb{L}$. По аналогии с леммой Пуанкаре положим

$$
\lambda(m, \mathbf{u})=\int_{0}^{1} \sum_{j} u^{j} \chi_{j}(m, \tau \mathbf{u}) d \tau \quad \text { для всех } \quad m \in \mathbb{L}, \quad \mathbf{u} \in \mathbf{V} .
$$

Тогда

$$
\begin{aligned}
\delta_{u^{i}} \lambda & =\sum_{n} T_{-n} \partial_{u_{n}^{i}} \lambda=\int_{0}^{1}\left(\chi_{i}(m, \tau \mathbf{u})+\sum_{j, n}\left(T_{-n} u^{j}\right)\left(T_{-n} \partial_{u_{n}^{i}} \chi_{j}\right)(m, \tau \mathbf{u}) \tau\right) d \tau= \\
& =\int_{0}^{1}\left(\chi_{i}(m, \tau \mathbf{u})+\tau \sum_{j, n} u_{n}^{j}\left(\partial_{u^{i}} T_{n} \chi_{j}\right)(m, \tau \mathbf{u})\right) d \tau= \\
& =\int_{0}^{1}\left(\chi_{i}(m, \tau \mathbf{u})+\tau \sum_{j, n} u_{n}^{j}\left(\partial_{u_{n}^{j}} \chi_{i}\right)(m, \tau \mathbf{u})\right) d \tau= \\
& =\int_{0}^{1} \frac{\partial}{\partial \tau}\left(\tau \chi_{i}(m, \tau \mathbf{u})\right) d \tau=\left.\left(\tau \chi_{i}(m, \tau \mathbf{u})\right)\right|_{\tau=0} ^{\tau=1}=\chi_{i}(m, \mathbf{u}), \quad i \in I .
\end{aligned}
$$

Каждая функция $f=\left(f^{i}\right) \in \mathcal{E}$ задает два линейных отображения:

1) $f_{*}: \mathcal{E} \rightarrow \mathcal{E}, g \mapsto f_{*}(g)$, где $f_{*}(g)^{i}=\operatorname{ev}_{g}\left(f^{i}\right)=\sum_{j, n}\left(\partial_{u_{n}^{j}} f^{i}\right) T_{n} g^{j}$

2) $f^{*}: \mathcal{E}^{*} \rightarrow \mathcal{E}^{*}, \chi \mapsto f^{*}(\chi)$, где $f^{*}(\chi)_{j}=\sum_{i, n} T_{-n}\left(\left(\partial_{u_{n}^{j}} f^{i}\right) \chi_{i}\right)$.

ПРЕДЛОЖЕНИЕ 11. Отображения $f_{*} u f^{*}$ сопряжены по Лагранжу:

$$
\left.\sum_{i, m}\left(\chi_{i} f_{*}(g)^{i}\right)\right|_{\mathbf{j}(\phi)}(m)=\left.\sum_{j, m}\left(f^{*}(\chi)_{j} g^{j}\right)\right|_{\mathbf{j}(\phi)}(m)
$$

для всех $\chi \in \mathcal{E}^{*}, g \in \mathcal{E}, \phi \in \operatorname{Map}(\mathbb{L} ; V)$.

Доказательство аналогично доказательству предложения 9.

ПрЕДЛОЖЕНИЕ 12. Коммутатор

$$
\left[\delta_{u^{i}}, \mathrm{ev}_{f}\right]=\sum_{j}\left(f^{*}\right)_{i}^{j} \circ \delta_{u^{j}} \quad \text { для всех } \quad i \in I,
$$

где $\left(f^{*}\right)_{i}^{j}=\sum_{n} T_{-n} \circ\left(\partial_{u_{n}^{i}} f^{j}\right)$.

ДокАЗАТЕЛЬСтво. С учетом предложения 3 имеем

$$
\begin{aligned}
\delta_{u^{i}} \circ \mathrm{ev}_{f} & =\partial_{u^{i}} \circ \sum_{m} T_{-m} \circ \mathrm{ev}_{f}=\partial_{u^{i}} \circ \mathrm{ev}_{f} \circ \sum_{m} T_{-m}= \\
& =\sum_{j, n} \partial_{u^{i}} \circ\left(T_{n} f^{j}\right) \circ \partial_{u_{n}^{j}} \circ \sum_{m} T_{-m}= \\
& =\sum_{j, n}\left(\left(\partial_{u^{i}} T_{n} f^{j}\right)+\left(T_{n} f^{j}\right) \circ \partial_{u^{i}}\right) \circ \partial_{u_{n}^{j}} \circ \sum_{m} T_{-m}=
\end{aligned}
$$




$$
\begin{aligned}
& =\sum_{j, n}\left(T_{n} \circ\left(\partial_{u_{-n}^{i}} f^{j}\right) \circ T_{-n} \circ \partial_{u_{n}^{j}}+\left(T_{n} f^{j}\right) \circ \partial_{u_{n}^{j}} \circ \partial_{u^{i}}\right) \circ \sum_{m} T_{-m}= \\
& =\sum_{j, n}\left(T_{-n} \circ\left(\partial_{u_{n}^{i}} f^{j}\right) \circ \partial_{u^{j}} \circ \sum_{m} T_{n-m}+\left(T_{n} f^{j}\right) \circ \partial_{u_{n}^{j}} \circ \partial_{u^{i}} \circ \sum_{m} T_{-m}\right)= \\
& =\sum_{j}\left(f^{*}\right)_{i}^{j} \circ \delta_{u^{j}}+\operatorname{ev}_{f} \circ \delta_{u^{i}} \quad \text { для всех } \quad i \in I .
\end{aligned}
$$

\section{3. ЭВОЛЮЦИОННЫЕ СИСТЕМЫ НА РЕШЕТКЕ}

3.1. Эволюционные системы. Введем новую независимую переменную $t \in \mathbb{R}$ и новые пространства:

1) $\mathcal{N}=\mathcal{N}(\mathbb{R} \times \mathbb{L} \times \mathbf{V} ; W)=\operatorname{Map}\left(\mathbb{L} ; \mathcal{C}^{\infty}(\mathbb{R} \times \mathbf{V} ; W)\right)$;

2) $\mathcal{B}=\mathcal{B}(\mathbb{R} \times \mathbb{L} \times \mathbf{V})=\operatorname{Map}\left(\mathbb{L} ; \mathcal{C}^{\infty}(\mathbb{R} \times \mathbf{V})\right)$;

3) $\mathcal{F}=\mathcal{N}(\mathbb{R} \times \mathbb{L} \times \mathbf{V} ; V)=\operatorname{Map}\left(\mathbb{L} ; \mathcal{C}^{\infty}(\mathbb{R} \times \mathbf{V} ; V)\right)$;

4) $\mathcal{F}^{*}=\mathcal{N}\left(\mathbb{R} \times \mathbb{L} \times \mathbf{V} ; V^{*}\right)=\operatorname{Map}\left(\mathbb{L} ; \mathcal{C}^{\infty}\left(\mathbb{R} \times \mathbf{V} ; V^{*}\right)\right)$;

5) $\Phi=\Phi(\mathbb{R} \times \mathbb{L} ; V)=\operatorname{Map}\left(\mathbb{L} ; \mathcal{C}^{\infty}(\mathbb{R} ; V)\right)$, где

$$
\operatorname{Map}\left(\mathbb{L} ; \mathcal{C}^{\infty}(\mathbb{R} \times \mathbf{V} ; W)\right)=\operatorname{limind}_{p \rightarrow \infty} \operatorname{Map}\left(\mathbb{L} ; \mathcal{C}^{\infty}\left(\mathbb{R} \times \mathbf{V}^{p} ; W\right)\right)
$$

Ради простоты обозначений, для пары функций $f=f(t, m, \mathbf{u}) \in \mathcal{N}$ и $\phi=$ $\phi(t, m) \in \operatorname{Map}\left(\mathbb{L} ; \mathcal{C}^{\infty}(\mathbb{R} ; \mathbf{V})\right)$ будем писать

$$
\left.f\right|_{\phi}=\left.f\right|_{\phi}(t, m)=f(t, m, \phi(t, m)) \in \operatorname{Map}\left(\mathbb{L} ; \mathcal{C}^{\infty}(\mathbb{R} ; W)\right) .
$$

Введенные выше вариационные операции действуют и на пространствах $\mathcal{N}, \mathcal{B}, \mathcal{F}$, $\mathcal{F}^{*}$ и $\Phi$, переменная $t$ является в этой ситуации параметром.

Объект нашего изучения - дифференциально-разностные системы уравнений

$$
\partial_{t} u^{i}=f^{i}(t, m, \mathbf{u}), \quad i \in I,
$$

коротко $\partial_{t} u=f$, где функция $f \in \mathcal{F}, \partial_{t}-$ частная производная по $t$. Решения этой системы - функции $\phi=\phi(t, m) \in \Phi$ такие, что

$$
\partial_{t} \phi^{i}(t, m)=f^{i}(t, m, \mathbf{j}(\phi)(t, m)) \quad \text { для всех } \quad t \in \mathbb{R}, \quad m \in \mathbb{L}, \quad i \in I,
$$

коротко $\partial_{t} \phi=\left.f\right|_{\mathbf{j}(\phi)}$.

ПРеДЛОЖЕНИЕ 13. Пусть $\phi \in \Phi$ есть решение системы $\partial_{t} u=f$. Тогда для любого $n \in \mathbb{L}$ функция $\phi_{n}=T_{n} \phi \in \Phi$ есть решение сдвинутой системы $\partial_{t} u_{n}=T_{n} f$, m.e. $\partial_{t}\left(T_{n} \phi\right)=\left.\left(T_{n} f\right)\right|_{\mathbf{j}(\phi)}$.

ДокАзАтЕльство. Действительно, в силу предложения 2

$$
\partial_{t}\left(T_{n} \phi\right)=T_{n}\left(\partial_{t} \phi\right)=T_{n}\left(\left.f\right|_{\mathbf{j}(\phi)}\right)=\left.\left(T_{n} f\right)\right|_{\mathbf{j}(\phi)}
$$


С каждой системой $u_{t}=f$ ассоциируется оператор полной эволюиии

$$
\mathrm{Ev}_{f}=\partial_{t}+\mathrm{ev}_{f}=\partial_{t}+\sum_{i, n}\left(T_{n} f^{i}\right) \partial_{u_{n}^{i}}
$$

ПреДЛОЖЕНИЕ 14. Для данной функиии $\phi \in \Phi$ равенство

$$
\left.\left(\operatorname{Ev}_{f} F\right)\right|_{\mathbf{j}(\phi)}=\partial_{t}\left(\left.F\right|_{\mathbf{j}(\phi)}\right)
$$

имеет место для всех $F \in \mathcal{B}$ тогда и только тогда, когда ф есть решение системь $u_{t}=f$.

ДокАЗАтЕЛьство. Действительно, с одной стороны,

$$
\begin{aligned}
\left.\left(\operatorname{Ev}_{f} F\right)\right|_{\mathbf{j}(\phi)} & =\left.\left(\partial_{t} F\right)\right|_{\mathbf{j}(\phi)}+\left.\left.\sum_{i, n}\left(\partial_{u_{n}^{i}} F\right)\right|_{\mathbf{j}(\phi)}\left(T_{n} f^{i}\right)\right|_{\mathbf{j}(\phi)}= \\
& =\left.\left(\partial_{t} F\right)\right|_{\mathbf{j}(\phi)}+\left.\sum_{i, n}\left(\partial_{u_{n}^{i}} F\right)\right|_{\mathbf{j}(\phi)} T_{n}\left(\left.f^{i}\right|_{\mathbf{j}(\phi)}\right),
\end{aligned}
$$

а с другой стороны,

$$
\begin{aligned}
\partial_{t}\left(\left.F\right|_{\mathbf{j}(\phi)}\right) & =\left.\left(\partial_{t} F\right)\right|_{\mathbf{j}(\phi)}+\left.\sum_{i, n}\left(\partial_{u_{n}^{i}} F\right)\right|_{\mathbf{j}(\phi)} \partial_{t}\left(T_{n} \phi^{i}\right)= \\
& =\left.\left(\partial_{t} F\right)\right|_{\mathbf{j}(\phi)}+\left.\sum_{i, n}\left(\partial_{u_{n}^{i}} F\right)\right|_{\mathbf{j}(\phi)} T_{n}\left(\partial_{t} \phi^{i}\right) .
\end{aligned}
$$

Следовательно, требуемое равенство имеет место тогда и только тогда, когда $\left.f^{i}\right|_{\mathbf{j}(\phi)}$ $=\partial_{t} \phi^{i}$ для всех $i \in I$, т.е. когда $\phi$ есть решение системы $u_{t}=f$.

3.2. Симметрии. Согласно общим принципам алгебро-геометрического подхода (группового анализа) [1], [3], [5], [6] (инфинитезимальные) симметрии данной эволюционной системы $\partial_{t} u=f$ суть эволюционные дифференцирования, коммутирующие с оператором полной эволюции $\mathrm{Ev}_{f}$. Подробнее, функция $g \in \mathcal{F}$ задает симметрию эволюиионной системы $\partial_{t} u=f$, если коммутатор $\left[\operatorname{Ev}_{f}, \mathrm{ev}_{g}\right]=0$.

Определим отображение $\operatorname{Ev}_{f}: \mathcal{F} \rightarrow \mathcal{F}$ по правилу: $\operatorname{Ev}_{f}(g)^{i}=\operatorname{Ev}_{f}\left(g^{i}\right)$ для всех $i \in I, g=\left(g^{i}\right) \in \mathcal{F}$.

ПреДЛОЖЕНИЕ 15. Функиия $g \in \mathcal{F}$ задает симметрию эволюиионной системы $\partial_{t} u=f$ тогда и только тогда, когда она удовлетворяет системе уравнений

$$
\left(\mathrm{Ev}_{f}-f_{*}\right)(g)=0 .
$$

ДокАЗАтЕльство. Действительно, коммутатор

$$
\begin{aligned}
{\left[\operatorname{Ev}_{f}, \mathrm{ev}_{g}\right] } & =\sum_{i, m}\left(\operatorname{Ev}_{f}\left(T_{m} g^{i}\right)-\operatorname{ev}_{g}\left(T_{m} f^{i}\right)\right) \partial_{u_{m}^{i}}= \\
& =\sum_{i, m} T_{m}\left(\operatorname{Ev}_{f}\left(g^{i}\right)-\operatorname{ev}_{g}\left(f^{i}\right)\right) \partial_{u_{m}^{i}}
\end{aligned}
$$

Остается лишь воспользоваться определениями отображений $\operatorname{Ev}_{f}$ и $f_{*}$. 
3.3. Законы сохранения. Как известно (см., например, [7]), пара $(\varrho, J) \in$ $\mathcal{B}^{1+D}$, где плотность $\varrho \in \mathcal{B}$, вектор $J=\left(J^{\alpha}\right) \in \mathcal{B}^{D}$ (напомним, что $D$ - размерность решетки $\mathbb{L}$ ), называется сохраняющимся током для системы $\partial_{t} u=f$, если она удовлетворяет уравнению неразрывности

$$
\operatorname{Ev}_{f} \varrho+\Delta \operatorname{iv} J=0 .
$$

ПРЕДЛОЖЕНИЕ 16. Пусть $(\varrho, J)$ - сохраняющийся ток, а ф-решение системъ $\partial_{t} u=f$. Если функиии $\left.\varrho\right|_{\mathbf{j}(\phi)},\left.J^{1}\right|_{\mathbf{j}(\phi)}, \ldots,\left.J^{D}\right|_{\mathbf{j}(\phi)}$ суммируемые, то

$$
\partial_{t}\left(\left.\sum_{m} \varrho\right|_{\mathbf{j}(\phi)}(t, m)\right)=0
$$

Другими словами, функиионал $\mathrm{R}(\phi)(t)=\left.\sum_{m} \varrho\right|_{\mathbf{j}(\phi)}(t, m)$ сохраняется на решениях.

ДокАзАТЕльство. Действительно, в силу предложения 14

$$
\left.\partial_{t} \sum_{m \in \mathbb{L}} \varrho\right|_{\mathbf{j}(\phi)}(t, m)=\sum_{m \in \mathbb{L}} \partial_{t}\left(\left.\varrho\right|_{\mathbf{j}(\phi)}(t, m)\right)=\left.\sum_{m \in \mathbb{L}}\left(\operatorname{Ev}_{f} \varrho\right)\right|_{\mathbf{j}(\phi)}(t, m)
$$

в то время как в силу предложений 2 и 4

$$
\left.\sum_{m \in \mathbb{L}}\left(\Delta_{\alpha} J^{\alpha}\right)\right|_{\mathbf{j}(\phi)}(t, m)=\sum_{m \in \mathbb{L}} \Delta_{\alpha}\left(\left.J^{\alpha}\right|_{\mathbf{j}(\phi)}(t, m)\right)=0 \quad \text { для всех } \quad 1 \leqslant \alpha \leqslant D,
$$

так что достаточно воспользоваться уравнением неразрывности.

Токи $(\varrho, J)$ вида

$$
\varrho=\Delta \operatorname{iv} A=\sum_{\alpha} \Delta_{\alpha} A^{\alpha}, \quad J^{\alpha}=-\operatorname{Ev}_{f} A^{\alpha}+\sum_{\beta} \Delta_{\beta} B^{\alpha \beta}
$$

где $A^{\alpha}, B^{\alpha \beta} \in \mathcal{B}, B^{\alpha \beta}+B^{\beta \alpha}=0,1 \leqslant \beta, \alpha \leqslant D$, называются тривиальными, поскольку в этом случае функционал $\mathrm{R}(\phi)=0$ для всех функций $\phi \in \Phi$ в силу предложений 2 и 4 . Для данной системы $\partial_{t} u=f$ множество $\mathcal{C C}_{f}$ всех сохраняющихся токов является линейным пространством, а множество всех тривиальных токов $\mathcal{T} \mathcal{C C}_{f}-$ его подпространством, так что определено факторпространство $\mathcal{C} \mathcal{L}_{f}=\mathcal{C C}_{f} / \mathcal{T C C}_{f}$ законов сохранения данной системы.

Определено линейное отображение

$$
\text { char: } \mathcal{C C}_{f} \rightarrow \mathcal{F}^{*}, \quad(\varrho, J) \mapsto \delta_{u} \varrho,
$$

где величина $\chi=\delta_{u} \varrho$ называется характеристикой тока $(\varrho, J)$.

ПрЕДЛОЖЕНИЕ 17. Ток $(\varrho, J) \in \mathcal{T C C}_{f}$ тогда и толъко тогда, когда его характеристика $\delta_{u} \varrho=0$. Иначе говоря, ядро Ker char $=\mathcal{T C C}_{f}$.

ДокАЗАтельство. Пусть ток $(\varrho, J)$ тривиальный, так что $\varrho=\Delta \mathrm{iv} A$, где $A \in \mathcal{B}^{D}$. Тогда согласно предложению $7 \delta_{u} \varrho=\delta_{u}(\Delta \mathrm{iv} A)=0$. Если $(\varrho, J) \in \mathcal{C C}_{f}$ и $\delta_{u} \varrho=0$, 
то в силу того же предложения $7 \varrho=\Delta \mathrm{iv} A, A \in \mathcal{B}^{D}$. Положим $\tilde{J}^{\alpha}=J^{\alpha}+\operatorname{Ev}_{f} A^{\alpha}$, $1 \leqslant \alpha \leqslant D$. Тогда

$$
\Delta \mathrm{iv} \tilde{J}=\Delta \mathrm{iv} J+\sum_{\alpha} \Delta_{\alpha}\left(\operatorname{Ev}_{f} A^{\alpha}\right)=\Delta \operatorname{iv} J+\operatorname{Ev}_{f}(\Delta \operatorname{iv} A)=\Delta \operatorname{iv} J+\operatorname{Ev}_{f} \varrho=0,
$$

так как коммутатор $\left[\Delta_{\alpha}, \mathrm{Ev}_{f}\right]=0,1 \leqslant \alpha \leqslant D$, и имеет место уравнение неразрывности для тока $(\varrho, J)$. Согласно предложению $8 \tilde{J}^{\alpha}=\sum_{\beta} \Delta_{\beta} B^{\alpha \beta}, B^{\alpha \beta}+B^{\beta \alpha}=0$, $1 \leqslant \beta, \alpha \leqslant D$, и, значит, ток $(\varrho, J)$ тривиальный.

СледСтвиЕ. Отображение char: $\mathcal{C C}_{f} \rightarrow \mathcal{F}^{*}$ индуиирует мономорфизм (линейное отображсение с тривиальным ядром)

$$
\text { Char: } \mathcal{C} \mathcal{L}_{f} \rightarrow \mathcal{F}^{*}, \quad[(\varrho, J)] \mapsto \delta_{u} \varrho .
$$

Определим отображение $\operatorname{Ev}_{f}: \mathcal{F}^{*} \rightarrow \mathcal{F}^{*}$ по правилу $\operatorname{Ev}_{f}(\chi)_{i}=\operatorname{Ev}_{f}\left(\chi_{i}\right)$ для всех $i \in I, \chi=\left(\chi_{i}\right) \in \mathcal{F}$.

ПреДЛОЖЕНИЕ 18. Функиия $\chi \in \mathcal{F}^{*}$ есть характеристика некоторого тока $(\varrho, J) \in \mathcal{C C}_{f}$ тогда и только тогда, когда $\chi_{*}=\chi^{*} u\left(\operatorname{Ev}_{f}+f^{*}\right) \chi=0$. Другими словами, образ

$$
\operatorname{Im} \text { char }=\operatorname{Im} \text { Char }=\left\{\chi \in \mathcal{F}^{*} ; \chi_{*}=\chi^{*},\left(\operatorname{Ev}_{f}+f^{*}\right) \chi=0\right\} .
$$

ДокАЗАТЕЛЬСтво. Согласно предложению 10 функция $\chi=\delta_{u} \varrho$ тогда и только тогда, когда $\chi_{*}=\chi^{*}$. Далее, пусть $(\varrho, J) \in \mathcal{C C}_{f}$ и $\chi=\delta_{u} \varrho$, тогда в силу предложений 12 и 7

$$
\begin{aligned}
\left(\operatorname{Ev}_{f}+f^{*}\right)(\chi)_{i} & =\partial_{t}\left(\delta_{u^{i}} \varrho\right)+\operatorname{ev}_{f}\left(\delta_{u^{i}} \varrho\right)+\sum_{j}\left(f^{*}\right)_{i}^{j}\left(\delta_{u^{j}} \varrho\right)= \\
& =\delta_{u^{i}}\left(\partial_{t} \varrho+\operatorname{ev}_{f} \varrho\right)=\delta_{u^{i}}\left(\operatorname{Ev}_{f} \varrho\right)=-\delta_{u^{i}}(\Delta \operatorname{iv} J)=0, \quad i \in I .
\end{aligned}
$$

Наоборот, пусть $\chi=\delta_{u} \varrho$ и $\left(\mathrm{Ev}_{f}+f^{*}\right) \chi=0$, тогда аналогичным образом имеем

$$
\left(\operatorname{Ev}_{f}+f^{*}\right)\left(\delta_{u} \varrho\right)=\delta_{u}\left(\operatorname{Ev}_{f} \varrho\right)=0 .
$$

В силу предложения $7 \operatorname{Ev}_{f} \varrho=-\Delta \operatorname{iv} J$ для некоторого $J \in \mathcal{B}^{D}$, так что пара $(\varrho, J) \in$ $\mathcal{C C}_{f}$

Таким образом, доказана

ТеоремА. Имеет место изоморфизм линейнъх пространств

$$
\text { Char: } \mathcal{C} \mathcal{L}_{f} \simeq\left\{\chi \in \mathcal{F}^{*} ; \chi_{*}=\chi^{*},\left(\operatorname{Ev}_{f}+f^{*}\right) \chi=0\right\} .
$$

ЗАмЕчАниЕ 5. По данной характеристике $\chi$ плотность $\varrho$ восстанавливается по формуле (см. доказательство предложения 10)

$$
\varrho(t, m, \mathbf{u})=\int_{0}^{1} \sum_{j} u^{j} \chi_{j}(t, m, \tau \mathbf{u}) d \tau, \quad t \in \mathbb{R}, \quad m \in \mathbb{L}, \quad \mathbf{u} \in \mathbf{V} .
$$

Вектор тока $J$ в приложениях играет второстепенную роль, но в случае необходимости его можно восстановить с помощью предложения 7. 
Благодарности. Работа выполнена при финансовой поддержке РФФИ (грант № 07-01-00144-а) и Программы поддержки ведущих научных школ (грант НШ$3224.2008 .1)$.

\section{Список литературы}

[1] В. В. Жаринов, ТМФ, 68:2 (1986), 163-171.

[2] Л. В. Овсянников, Групповой анализ дифференииальных уравнений, Наука, М., 1978.

[3] Н. Х. Ибрагимов, Группь преобразований в математической физике, Наука, М., 1983.

[4] П. Олвер, Приложения групп Ли к дифференциальным уравнениям, Мир, М., 1989.

[5] V.V. Zharinov, Lecture Notes on Geometrical Aspects of Partial Differential Equations, Ser. Sov. and East Eur. Math., 9, World Scientific, Singapore, 1992.

[6] А. М. Виноградов, И. С. Красильщик (ред.), Симметрии и законы сохранения уравнений математической физики, Факториал, М., 1997.

[7] W. Hereman, M. Colagrosso, R. Sayers et al., "Continuous and discrete homotopy operators and the computation of conservation laws", Differential Equations with Symbolic Computation, Trends Math., eds. D. Wang, Z. Zheng, Birkhäuser, 2005, 255-290.

[8] P. E. Hydon, E. L. Mansfield, Found. Comput. Math., 4:2 (2004), 187-217.

[9] В.Э. Адлер, А. Б. Шабат, Р. И. Ямилов, ТМФ, 125:3 (2000), 355-424.

[10] I.M. Anderson, The variational bicomplex, http://www.math.usu.edu/ ${ }^{\sim g}$ g_mp/ Publications/VB/vb.pdf, 2004. 